\title{
CORRESPONDENCE
}

\section{Emerging SARS-CoV-2 variants reduce neutralization sensitivity to convalescent sera and monoclonal antibodies}

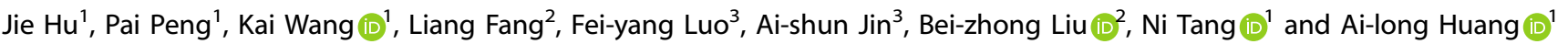 \\ Cellular \& Molecular Immunology (2021) 18:1061-1063; https://doi.org/10.1038/s41423-021-00648-1
}

Coronaviruses are enveloped, positive-stranded RNA viruses that contain the largest known RNA genomes to date. As severe acute respiratory syndrome coronavirus 2 (SARS-CoV-2) continues to circulate in the human population, multiple mutations have accumulated over time, which may affect its transmission, virulence and antigenicity. Neutralizing antibodies (NAbs) elicited by natural infection or vaccination are likely to be a key immune correlate for protection against SARS-CoV-2 infection. A decline in the antibody response to SARS-CoV-2 in convalescent individuals and reinfections by different viral variants have been reported. ${ }^{1-3}$ It is therefore important to gain insights into the infectivity and antigenicity of SARS-CoV-2 variants.

Spike-specific antibodies contribute the majority of the neutralizing activity in most convalescent human sera. Two SARS-CoV-2 variants, N501Y.V1 (also known as B.1.1.7 lineage or VOC-202012/01) and N501Y.V2 (B.1.351 lineage), reported from the United Kingdom and South Africa, respectively, contain several mutations in the receptor-binding domain (RBD) of Spike and are of particular concern. To address the infectivity and neutralization escape phenotypes potentially caused by these mutations, we used the SARS-CoV-2 pseudovirus system to compare the viral infectivity as well as the neutralization activities of convalescent sera and monoclonal antibodies (mAbs) against SARS-CoV-2 variants.

The blood samples $(n=40)$ of 20 patients with COVID-19 obtained in February and October 2020 in Chongqing were previously described. ${ }^{2}$ Eight RBD-specific mAbs with neutralizing capability against SARS-CoV-2 were obtained from the blood samples of COVID-19 convalescent patients. ${ }^{4}$ DNA sequences encoding the reference strain wild-type (WT) and mutant Spike proteins of SARS-CoV-2 were codon-optimized and synthesized by Sino Biological Inc. (Beijing, China) and GenScript Inc. (Nanjing, China). Using a luciferase-expressing lentiviral pseudotype system, we expressed WT and N501Y.V1 (variant 1) and N501Y.V2 (variant 2) mutant Spike proteins in enveloped virions. NAbs were measured by pseudovirus-based assays in 293T-ACE2 cells. The inhibitory dose $\left(\mathrm{ID}_{50}\right)$ was calculated based on the titers of NAbs.

First, the infectivity of pseudotyped viral particles was measured by luciferase assay as previously described. ${ }^{5}$ As shown in Fig. 1a, the entry efficiencies of Spike pseudotyped viruses bearing the $\mathrm{N} 501 \mathrm{Y}$ variant 1 or variant 2 mutant were $\sim 3$ to 4.4 times higher than that of the WT pseudovirus when viral input was normalized, suggesting that these spike variants promote the infectivity of
SARS-CoV-2. Then, we assessed the neutralizing efficacy of 40 convalescent serum samples from 20 individuals at two time points with a pseudovirus neutralization assay. At follow-up time point 1 , corresponding to a median of 25 days (range 5-33 days) post symptom onset, most sera were significantly less effective than the WT pseudovirus in neutralizing the N501Y variant 1 and variant 2 (Fig. 1b). The mean NAb titers were 825 for WT, 343 for variant 1, and 148 for variant 2 . The neutralizing activity of two samples against N501Y.V1 was reduced by greater than tenfold. Notably, the NAb titers of six samples (30\%) decreased below the threshold against variant 2 (Fig. 1b). At $\sim 8$ months post symptom onset (follow-up time point 2), 17 samples of 20 participants (85\%) retained titers of $I D_{50}>40$ against WT pseudovirus, whereas the NAb titers of 8 samples $(40 \%)$ and 18 samples $(90 \%)$ decreased below the threshold against N501Y variant 1 and variant 2, respectively (Fig. 1c). These data indicate that N501Y variant 1 and variant 2 escape neutralizing antibodies in some COVID-19 convalescent sera.

In addition, we assessed the impact of these variants on the neutralizing activity of human mAbs isolated from COVID-19 convalescent patients. All eight antibodies potently neutralized the WT pseudovirus, while two mAbs (CQ016 and CQ045) were only minimally affected by the variants. However, the neutralization activities of the six other mAbs were reduced or abolished by either N501Y variant 1 or variant 2 (Fig. 1d). Among them, three mAbs were threefold or more less effective against N501Y.V1 and five against N501Y.V2 (Fig. 1d). Notably, two mAbs (CQ026 and CQ038) showed no neutralizing activity against N501Y.V2. Moreover, variant 2 reduced the neutralization sensitivity with CQ046 by 26-fold, which showed the most potent activity against WT pseudovirus. The $\mathrm{IC}_{50}$ of the $\mathrm{mAb}$ CQ046 increased from $7.4 \mathrm{ng} / \mathrm{ml}$ (WT) to $194 \mathrm{ng} / \mathrm{ml}$ (variant 2) (Fig. 1e). Overall, both N501Y variant 1 and variant 2 reduced neutralization sensitivity to most mAbs tested, while N501Y.V2 even abrogated the neutralizing activity of two mAbs.

Our findings indicated that N501Y variant 1 and variant 2 increase viral infectivity compared to that of the reference strain in vitro. Notably, both N501Y variant 1 and variant 2 contain the D614G and N501Y mutations in the Spike protein. The findings that variant 1 and variant 2 enhanced the infectivity of SARS-CoV2 in vitro are highly consistent with previous studies, which demonstrated that D614G and N501Y mutations enhanced the fitness and transmissibility of the virus, as evidenced by structure

\footnotetext{
'Key Laboratory of Molecular Biology for Infectious Diseases (Ministry of Education), Institute for Viral Hepatitis, Department of Infectious Diseases, the Second Affiliated Hospital, Chongqing Medical University, Chongqing, China; ${ }^{2}$ Yong-Chuan Hospital, Chongqing Medical University, Chongqing, China and ${ }^{3}$ Department of Immunology, College of Basic Medicine, Chongqing Medical University, Chongqing, China

Correspondence: Bei-zhong Liu (liubeizhong@cqmu.edu.cn) or Ni Tang (nitang@cqmu.edu.cn) or Ai-long Huang (ahuang@cqmu.edu.cn)

These authors contributed equally: Jie Hu, Pai Peng, Kai Wang.
}

Received: 24 January 2021 Accepted: 29 January 2021

Published online: 25 February 2021 
a
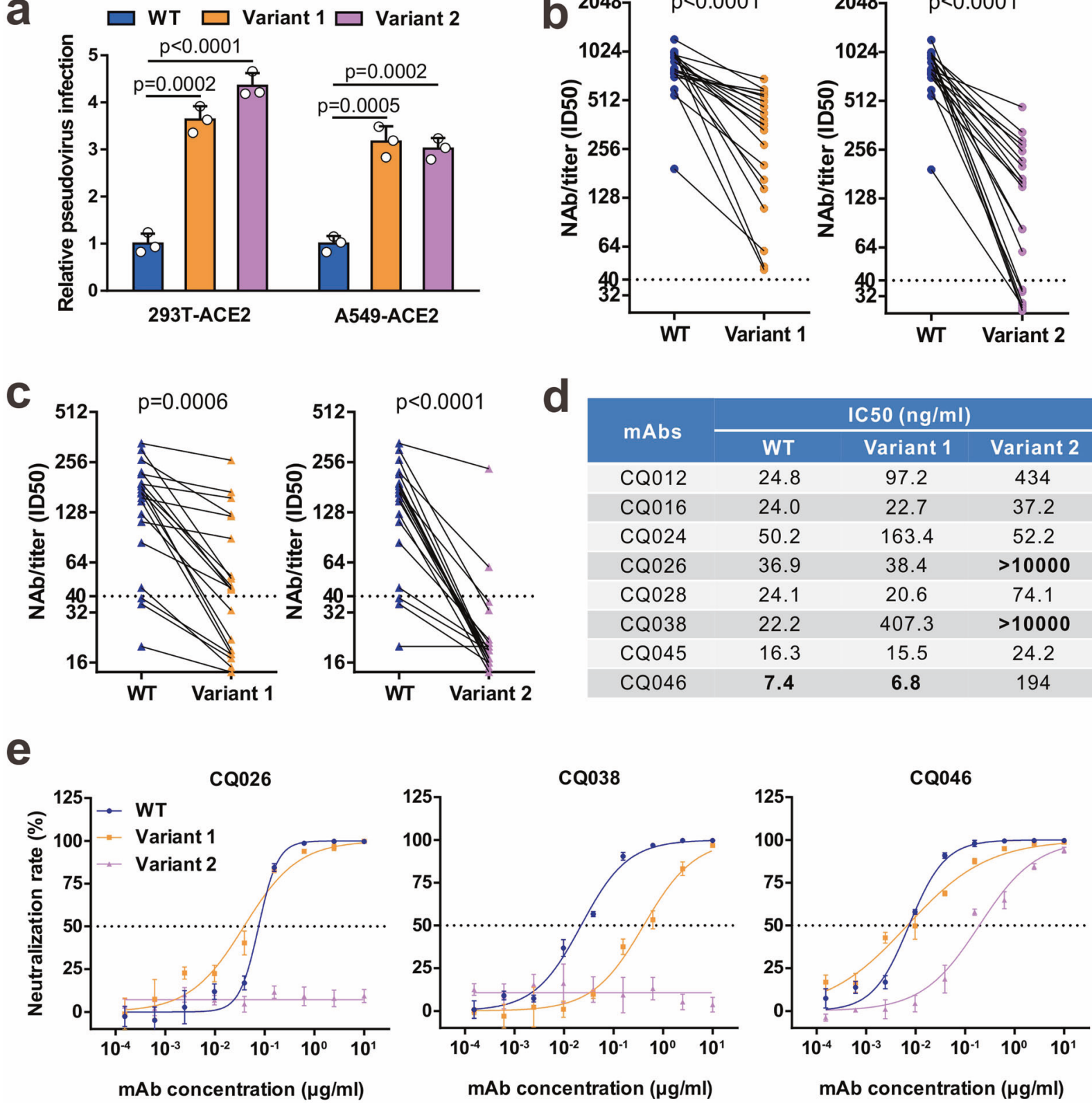

Fig. 1 Neutralizing activities of convalescent sera and monoclonal antibodies against SARS-CoV-2 variants. a Infectivity of WT and variant pseudoviruses assessed in 293T-ACE2 and A549-ACE2 cells. Cells were inoculated with equivalent doses of each pseudotyped virus. WT, wildtype Spike (GenBank: QHD43416) pseudotyped virus; Variant 1, N501Y.V1 mutant Spike pseudotyped virus (containing the H60/V70 and Y144 deletions and N501Y, A570D, D614G, P681H, T716I, S982A, and D1118H mutations); Variant 2, N501Y.V2 mutant Spike pseudotyped virus (containing the K417N, E484K, N501Y, and D614G mutations). Neutralization of WT and variant pseudoviruses by convalescent sera. Pseudovirus-based neutralization assays were performed to detect neutralizing antibody (NAb) titers against SARS-CoV-2. The thresholds of detection were 1:40 of the $\mathrm{ID}_{50}$. Twenty sera (indicated by circles) were drawn 5 to 33 days post symptom onset (b); 20 sera (indicated by triangles) were drawn $\sim 8$ months post symptom onset (c). The half-maximal inhibitory concentrations $\left(I_{50}\right)$ for tested monoclonal antibodies (mAbs) against pseudoviruses (d) and representative neutralization curves (e)

analysis and the increased number of clinical cases. ${ }^{6,7}$ Another key question is whether some mutations may enable immune evasion. It has been reported that neutralization escape mutants can be selected by passaging virus in the presence of NAbs. ${ }^{8}$ Consistent with other recent studies, ${ }^{9,10}$ we observed that two naturally occurring SARS-CoV-2 variants, N501Y variant 1 and variant 2, were more resistant than WT virus to neutralization by some mAbs and convalescent sera from patients who were infected in mid- to late- January 2020 when a "first-wave" virus was circulating in China. Consistently, Spike variants with the H60/V70 deletion or E484K mutation have significantly reduced susceptibility to neutralization by the polyclonal serum antibodies of some individuals. ${ }^{11,12}$ Whether these patients are at high risk of reinfection with 'second-wave' variants should be explored in further studies. It is also urgent to assess the effectiveness of currently authorized vaccines against these variants.

Collectively, this study will be helpful for understanding SARSCoV-2 infectivity and for the design of vaccines against COVID-19. Given the evolving nature of the SARS-CoV-2 RNA genome, antibody therapeutics and vaccine development require further considerations to accommodate mutations in Spike that may affect the antigenicity of the virus. Limitations of this study include 
its small sample size and the use of a nonreplicating pseudovirus system. Therefore, further studies with authentic SARS-CoV-2 viruses are required.

\section{ACKNOWLEDGEMENTS}

We would like to thank Prof. Cheguo Cai (Wuhan University, Wuhan, China) for providing the pNL4-3.Luc.R-E lentiviral plasmid. Our work has received funding support from the Emergency Project from the Science \& Technology Commission of Chongqing (cstc2020jscx-dxwtB0050, cstc2020jscx-fyzx0053), the Emergency Project for Novel Coronavirus Pneumonia from Chongqing Medical University (CQMUNCP0302), the Key Laboratory of Infectious Diseases (CQMU, 202005), the Leading Talent Program of CQ CSTC (CSTCCXLJRC201719), and a Major National Science \& Technology Program grant (2017ZX10202203) from the Science \& Technology Commission of China.

\section{AUTHOR CONTRIBUTIONS}

A.H., N.T., and B.L. developed the conceptual ideas and designed the study. J.H. and P.P. performed the experiments. L.F. and B.L. provided the samples. F.L. and A.J. were responsible for $\mathrm{mAb}$ purification. K.W. performed the statistical analysis. All authors provided scientific expertise and interpretation of the data for the work. K.W. drafted the manuscript. All authors have approved the final version of the manuscript.

\section{ADDITIONAL INFORMATION}

Supplementary information The online version contains supplementary material available at https://doi.org/10.1038/s41423-021-00648-1.

Competing interests: The authors declare no competing interests.

\section{REFERENCES}

1. Ibarrondo, F. J. et al. Rapid decay of anti-SARS-CoV-2 antibodies in persons with mild Covid-19. N. Engl. J. Med. 383, 1085-1087 (2020).

2. Peng, P. et al. Changes in the humoral immunity response in SARS-CoV-2 convalescent patients over 8 months. Cell. Mol. Immunol. 2021. https://doi.org/ 10.1038/s41423-020-00605-4.

3. Zhang, J. et al. COVID-19 reinfection in the presence of neutralizing antibodies. Natl Sci. Rev. 2021. https://doi.org/10.1093/nsr/nwab006.

4. Han, X. et al. A rapid and efficient screening system for neutralizing antibodies and its application for the discovery of potent neutralizing antibodies to SARSCoV-2 S-RBD. bioRxiv 2020. https://doi.org/10.1101/2020.08.19.253369.

5. Hu, J. et al. Development of cell-based pseudovirus entry assay to identify potential viral entry inhibitors and neutralizing antibodies against SARS-CoV-2. Genes Dis. 7, 551-557 (2020).

6. Korber, B. et al. Tracking changes in SARS-CoV-2 spike: evidence that D614G increases infectivity of the COVID-19 virus. Cell 182, 812-827.e19 (2020).

7. Starr, T. N. et al. Deep mutational scanning of SARS-CoV-2 receptor binding domain reveals constraints on folding and ACE2 binding. Cell 182, 1295-1310.e20 (2020).

8. Weisblum, Y. et al. Escape from neutralizing antibodies by SARS-CoV-2 spike protein variants. eLife. 9. https://doi.org/10.7554/eLife.61312.

9. Wibmer, C. K. et al. SARS-CoV-2 501Y.V2 escapes neutralization by South African COVID-19 donor plasma. bioRxiv 2021. https://doi.org/10.1101/2021.01. 18.427166.

10. Rees-Spear, C. et al. The impact of Spike mutations on SARS-CoV-2 neutralization. bioRxiv 2021. https://doi.org/10.1101/2021.01.15.426849.

11. Kemp, S. et al. Neutralising antibodies in Spike mediated SARS-CoV-2 adaptation. medRxiv 2020. https://doi.org/10.1101/2020.12.05.20241927.

12. Greaney, A. J. et al. Complete mapping of mutations to the SARS-CoV-2 spike receptor-binding domain that escape antibody recognition. Cell Host Microbe 29, 44-57.e9 (2021). 